\title{
Efecto de edulcorantes no calóricos en la calidad sensorial de jugo de naranja
}

\author{
Effect of non caloric sweeteners \\ in sensory quality of orange juice
}

\begin{abstract}
The consumption of non-caloric sweeteners is increasing in Chile being present in a wide variety of foods. The objective of this study was to evaluate the effect of distinct non-caloric sweeteners in the sensory qualities of orange juice as compared to sugar. The sweeteners selected were stevia, sucralose, saccharin and aspartame. These were added to natural juices in amounts equivalent to sugar in sweetness. Sensory analysis was performed with a group of youths of both sexes. An acceptability test was applied with an hedonic scale of 5 points evaluating aroma and flavor. The results indicate that the juice aroma is not affected by the use of sugar or sweeteners rated 3.5 to 3.7 (corresponding to "I like"). However, in the evaluation of flavor, significant differences were found, the best results corresponding to juice sweetened with sugar and sucralose (3,5 y 3,6 respectively).
\end{abstract}

Key words: sensorial analysis, non-nutritive sweeteners, sugar.

\section{INTRODUCCIÓN}

El sabor dulce juega un rol muy importante en los seres humanos, ya que la mayoría de las personas responden positivamente a la sensación de dulzor, y existe una propensión al consumo de alimentos dulces que se remonta a la vida temprana (1).

Los edulcorantes utilizados en la industria alimentaria se dividen en 2 grandes grupos: calóricos y no calóricos. Entre los edulcorantes no calóricos de mayor consumo se encuentran la sacarina de sodio cuyo poder edulcorante es 200 a 700 superior al azúcar, la sucralosa que es 600 veces más dulce que el azúcar, la estevia cuyo poder es 300 veces mayor que el azúcar y Aspartamo 200 veces más dulce que el azúcar $(2,3)$.

Actualmente, los edulcorantes no calóricos han ganado una mayor presencia en la alimentación, no tan sólo de personas diabéticas o con dietas hipocalóricas, si no que de personas sin patologías que han decidido disminuir el consumo del azúcar (sacarosa). Los edulcorantes no calóricos son utilizados como reemplazo total o parcial de la sacarosa; además, poseen un mayor poder endulzante que ésta (2).

Se discute que los edulcorantes no poseen un poder saciador como el azúcar, inclusive podrían causar la sensación
Vilma Quitral R. (1)

Anna Christina Pinheiro F. (1)

Constanza Carrera P. (2)

Gabriela Gallo U. (2)

Paola Moyano S. (2)

Julieta Salinas V. (2)

Paula Jimenez P. (1)

(1) Departamento de Nutrición, Facultad de Medicina Universidad de Chile. Santiago, Chile.

2) Escuela de Nutrición y Dietética, Facultad de Medicina, Universidad de Chile. Santiago, Chile.

Dirigir la correspondencia a:

Profesora

Vilma Quitral $R$

Departamento de Nutrición

Facultad de Medicina

Universidad de Chile

Teléfono: 562-9786241

E-mail:vquitral@med.uchile.cl

Este trabajo fue recibido el 16 de Mayo de 2014, aceptado con modificaciones el 16 de Agosto de 2014 y aceptado para ser publicado el 20 de Noviembre de 2014.

de hambre estimulando a comer en exceso, además podrían estimular los receptores del gusto, creando adicción al sabor dulce (4). Cada día el consumo de edulcorantes se hace más masivo en nuestra sociedad y están presentes en una gran variedad de alimentos. Los edulcorantes mantienen la palatabilidad de la dieta permitiendo reemplazar el azúcar de muchos alimentos y así reducir el aporte calórico de los mismos. La ingesta es segura, pero es necesario determinar la cantidad de edulcorantes en los alimentos y monitorear el consumo por la población, para determinar si están o no sobrepasando los IDA.

En un modelo animal en roedores, Swithers y cols. (5) establecieron que el libre acceso a edulcorantes no calóricos conduce a un aumento de peso corporal, adiposidad, e ingesta calórica. Resultados similares han sido encontrados en humanos ya que algunos estudios han demostrado una correlación directa entre el uso de edulcorantes artificiales y aumento de peso $(6,7)$. Sin embargo, otros estudios sugieren que el uso de edulcorantes artificiales pueden apoyar en la pérdida de peso (8) o no se han encontrado efectos concluyentes sobre su efecto en el IMC (9). Por otra parte, varias revisiones han concluido que existe evidencia que vincula el uso de edulcorantes artificiales con aumento de la ingesta de alimentos $(10,11)$. 
La Asociación Americana de Dietistas (12) entregó una posición favorable al uso de los edulcorantes de acuerdo a las regulaciones locales $y$, bajo la orientación de un profesional del área de la nutrición. Considera que pueden ser un potente aliado para aumentar la palatabilidad de frutas, vegetales y productos de panificación elaborados con granos enteros, aumentando su densidad de nutrientes y disminuyendo la densidad calórica. Otros autores indican que, hasta la fecha, no existe evidencia concluyente en relación a los efectos benéficos o adversos del uso de los edulcorantes artificiales no calóricos (3).

Para evaluar el grado de satisfacción de alimentos, con el fin de determinar cuál es el que presenta mayor aceptabilidad en una serie de productos, se utilizan pruebas sensoriales de respuesta subjetiva con consumidores, siendo las más aplicadas las pruebas hedónicas destinadas a medir cuánto agrada o desagrada un producto. Para estas pruebas se utilizan escalas categorizadas, que pueden tener diferente número de categorías y que comúnmente van desde el mínimo al máximo "agrado" $(13,14)$.

El objetivo de este estudio fue evaluar el efecto de distintos tipos de edulcorantes en la aceptabilidad del aroma y sabor del jugo de naranja.

\section{MATERIALES Y MÉTODOS}

Esta investigación correspondió a un estudio de tipo cualitativo, prospectivo y observacional. Se elaboraron jugos de naranja endulzados con azúcar, estevia, sucralosa, sacarina y aspartamo, en cantidades equivalentes de acuerdo al grado de dulzor descritas por cada proveedor. Para $200 \mathrm{ml}$ de jugo se adicionaron 10 gramos de azúcar (2 cucharaditas); en el caso de los jugos endulzados con estevia, se adicionaron 10 gotas $(0.5 \mathrm{ml})$; en jugos con sucralosa, se adicionaron 2 tabletas de $8 \mathrm{mg}$ cada una; en el caso de sacarina fueron 10 gotas $(0.5 \mathrm{ml})$ y con aspartamo se adicionaron 2 tabletas de $2 \mathrm{mg}$ cada una.

Para las pruebas de análisis sensorial, se conformó o un panel de consumidores compuesto por 59 estudiantes universitarios con edades entre 18 y 29 años, considerándose una probabilidad de error $\alpha$ de $1 \%$, probabilidad de error $\beta$ de $5 \%$, RMSL (error cuadrado medio dividido por la longitud de escala) de 0,30 y diferencia en las medias que se busca en el experimento de 0,2 (15). Los criterios de exclusión fueron padecer diabetes mellitus 1 o 2 y no consumir ningún producto con azúcar.

A los evaluadores se les presentaron 5 muestras de jugos de naranja endulzados (con azúcar, estevia, sucralosa, sacarina y aspartamo, respectivamente) en vasos de vidrio transparentes y cubiertos en la parte superior hasta el momento de la evaluación; la distribución de los jugos y la codificación de los vasos se realizaron al azar. Las evaluaciones sensoriales se realizaron en laboratorio con cabinas individuales aisladas y condiciones apropiadas para este propósito (16-18).

Los evaluadores procedieron a llenar sus datos en la ficha de respuesta (nombre, edad, fecha y hora), luego retiraron la cubierta de los vasos para evaluar el aroma de los jugos externamente a través de las fosas nasales y posteriormente evaluaron el sabor por medio de la degustación de cada jugo (19).

Para la evaluación sensorial se utilizó una prueba de aceptabilidad con escala hedónica de 5 puntos por parámetros, que califica desde 1 "me disgusta mucho" a 5 "me gusta mucho" (20) como se presenta en la figura 1.

Los resultados de los datos de características sensoriales percibidos por los evaluadores se presentan como promedios \pm desviación estándar. Para establecer si existen diferencias significativas entre las muestras se realizó un análisis de varianza (ANOVA), y en el caso de presentarse diferencias significativas $(p<0,05)$ se aplicó el test de DUNCAN de comparación múltiple o de rango múltiple. Para esto se utilizó el programa estadístico SPSS 15.0 para Windows.

\section{RESULTADOS}

La figura 2 presenta los resultados de la evaluación de aceptabilidad del aroma de los jugos con diferentes edulcorantes. Se observa que en todos los casos la aceptabilidad estuvo entre "no me gusta ni me disgusta" y "me gusta", no existiendo diferencias significativas entre los edulcorantes.

Al analizar los datos de acuerdo a sexo, se observó que los hombres calificaron levemente más bajo el aroma de los jugos. En el caso del jugo endulzado con aspartamo existieron diferencias significativas en la evaluación de hombres y mujeres

Escala hedónica de 5 puntos para evaluación sensorial de alimentos.

\begin{tabular}{|l|c|}
\hline \multicolumn{1}{|c|}{ Categoría } & Número \\
\hline Me disgusta mucho & 1 \\
\hline Me disgusta & 2 \\
\hline No me gusta ni me disgusta & 3 \\
\hline Me gusta & 4 \\
\hline Me gusta mucho & 5 \\
\hline
\end{tabular}


(datos no presentados).

La figura 3 presenta los resultados de la evaluación de aceptabilidad de sabor de los jugos con diferentes edulcorantes. Se observó que existe gran variabilidad según el edulcorante utilizado, con diferencias significativas. Los jugos endulzados con azúcar y sucralosa presentan mayor aceptabilidad, mientras que el jugo de menor aceptabilidad fue el endulzado con sacarina $(p<0.05)$.

\section{FIGURA 2}

Aceptabilidad de jugos de naranja con diferentes edulcorantes de acuerdo al aroma.

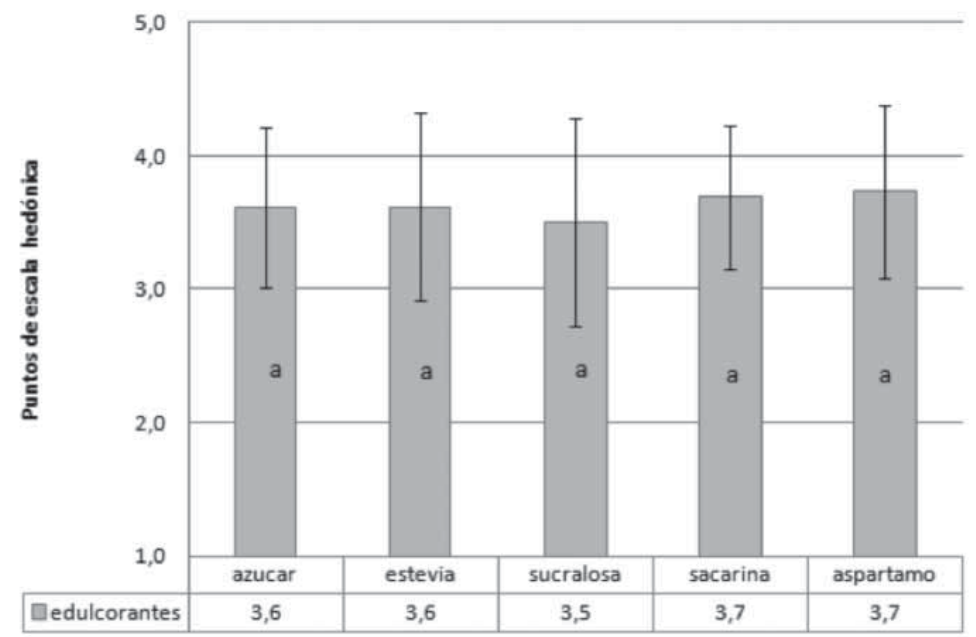

Letras distintas indican diferencias significativas $(p<0.05)$

FIGURA 3

Aceptabilidad de jugos de naranja con diferentes edulcorantes de acuerdo al sabor

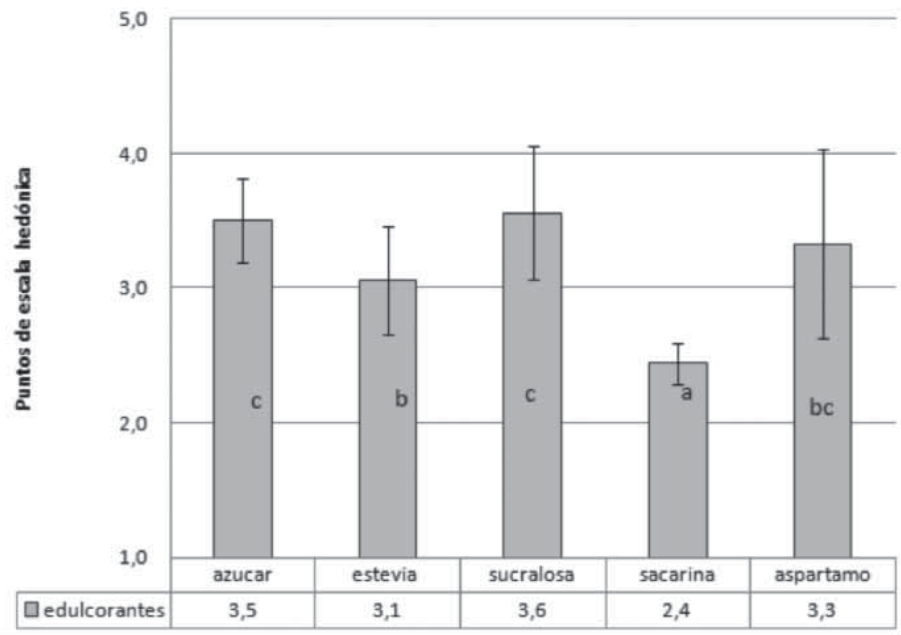

Letras distintas indican diferencias significativas $(p<0.05)$ 
Al realizar el análisis según sexo, los hombres manifestaron mayor aceptabilidad que las mujeres en los jugos con estevia, sacarina y sucralosa, aunque sin diferencias significativas; en el caso de los jugos con azúcar y aspartamo, las mujeres demostraron mayor aceptabilidad. La mayor puntuación en la escala hedónica la presentó el jugo con sucralosa evaluado por hombres y la menor corresponde al jugo con sacarina evaluado por mujeres.

\section{DISCUSIÓN}

En el presente estudio se demostró que el aroma de los jugos no se ve afectado por el tipo de edulcorante, lo que se contradice con los resultados de Da Porto y cols. (21), quienes determinaron el efecto de la adición de sacarosa, glucosa, fructosa, miel, jarabe de arce, acesulfame $K$, aspartamo y sorbitol sobre la formación de compuestos volátiles en licor de naranja; encontrando que sacarosa, glucosa, jarabe de arce y aspartamo aumentan la solubilidad de algunos compuestos volátiles que contribuyen al deseable aroma y sabor de naranja.

En cuanto a la diferencia por género, las mujeres manifestaron mayor aceptabilidad por el aroma de todos los jugos respecto a los hombres. De acuerdo a Choudhury y cols. (22) las mujeres generalmente son más sensibles y más hábiles para identificar los aromas que los hombres. Además, la actividad cerebral de las mujeres sobre la exposición a los olores es mucho más marcada $(23,24)$. En relación al sabor, Coonner y Booth (25) indicaron que los hombres muestran mayor preferencia por lo dulce que las mujeres, en promedio.

En nuestro estudio, los jugos endulzados con estevia, sacarina y aspartamo presentaron menor aceptabilidad respecto al jugo endulzado con azúcar, lo que concuerda con otras investigaciones $(26,27)$. Es importante considerar que los edulcorantes tienen características particulares en diferentes tipos de alimentos y bebidas y también pueden promover efectos indeseables como sabor residual o sabor amargo (28).

En un estudio sobre los efectos de los edulcorantes artificiales en la ingesta de alimentos y la saciedad, el aspartamo fue descrito por los participantes con mayor sabor agradable en comparación con estevia (29), lo que coincide con los resultados del presente estudio. Los jugos endulzados con estevia presentaron aceptabilidad significativamente menor que los endulzados con azúcar. De acuerdo a De Souza y cols. (30) la estevia es el mejor endulzante no calórico, ya que reduce el sabor amargo que otorgan otros edulcorantes, lo que se contradice con Cardoso y Bolini (28), quienes indican que la estevia presenta sabor amargo, especialmente en altas concentraciones.

Las muestras endulzadas con sacarina presentaron baja aceptabilidad, cercana a la categoría "me disgusta", sobre todo en mujeres. La evidencia indica que la sacarina es intensamente dulce, pero presenta un sabor residual caracterizado como amargo generalmente descrito por los consumidores como "sabor metálico" (31, 32).

Los jugos endulzados con sucralosa fueron bien evaluados en cuanto a su aceptabilidad, sin diferencias significativas respecto a los jugos endulzados con azúcar. Este resultado coincide con otras investigaciones ya que algunos estudios indican que la sucralosa ha sido considerado como el edulcorante que mejor sustituye la sacarosa, ya que provoca menos alteraciones sensoriales en los alimentos $(27,33,34)$. Posee un perfil de sabor muy similar al azúcar y no presenta sabor residual desagradable, característica indeseable que se encuentra en muchos otros edulcorantes no calóricos, como por ejemplo la sacarina. Además no es susceptible a cambios con altas temperaturas, pudiendo ser utilizado en preparaciones que son sometidas a altas temperaturas de cocción

En Chile, más del 60\% de la población mayor de 15 años presenta exceso de peso (35) y cerca del $21 \%$ de los niños que ingresan a la enseñanza básica son obesos (36). Aumentar la disponibilidad de productos industrializados libres de azúcares añadidos ha sido una medida de salud pública impulsada por varios países e incentivada por organismos internacionales como la Organización Mundial de la Salud. La evidencia indica una fuerte relación entre la ingesta de alimentos ricos en azúcares añadidos y la mayor prevalencia de trastornos asociados a la obesidad como es el caso de la hipertensión arterial e hipertrigliceridemia (37), hiperglicemia (38), aumento de masa grasa corporal (39), principalmente en la población joven.

Las medidas a adoptar en términos de políticas públicas podrían ser un aumento de los impuestos a las bebidas azucaradas (40) aunque pocos son los estudios que han podido evaluar el impacto de dichas medidas. Sin embargo, los análisis económicos han podido establecer que un aumento de los impuestos a las bebidas azucaradas pueden asociarse a una disminución de la ingesta energética y del IMC a largo plazo, principalmente entre consumidores jóvenes (40). En Chile, se trabaja en la adopción de mensajes de advertencia en el etiquetado nutricional de alimentos para aquellos productos que presentan altas concentraciones de azúcares, la que entra en vigencia a partir del 2014.

Para enfrentar el avance de la obesidad en la población es imprescindible poder contar con múltiples estrategias, las que deben contemplar la adopción de medidas estructurales como el aumento de las tasas impositivas para determinadas categorías de alimentos no saludables, así como una adaptación de la industria alimentaria a este nuevo escenario. Esta adaptación requiere necesariamente una mayor oferta de productos industrializados que puedan reemplazar aquellos productos tradicionales ricos en los nutrientes que potencialmente se encuentran asociados al mayor riesgo de enfermedades crónicas, como es el caso de los azúcares añadidos. Este estudio colabora con la generación de evidencia que puede apuntar a las mejores alternativas para el reemplazo de los azúcares añadidos en determinadas categorías de productos como es el caso de los jugos de frutas.

Solamente una sumatoria de acciones podrá entregar resultados alentadores a largo plazo en lo que se refiere a la disminución de la carga de enfermedades crónicas en la población.

\section{CONCLUSIÓN}

En base a los resultados obtenidos en este estudio, se concluye que la utilización de edulcorantes no calóricos no afecta el aroma del jugo de naranja, comparado con la utilización del azúcar. Sin embargo, en la evaluación de sabor, sí existen diferencias significativas. Las muestras con mayor aceptabilidad corresponden a jugos endulzados con azúcar y sucralosa.

\section{RESUMEN}

El consumo de edulcorantes no calóricos es cada vez más masivo en nuestro país y están presentes en una gran variedad de alimentos. El objetivo de este estudio fue evaluar el efecto de edulcorantes no calóricos en la calidad sensorial de jugo de naranja, en comparación con el azúcar. Los edulcorantes seleccionados fueron estevia, sucralosa, sacarina y aspartamo. Se adicionaron a jugos naturales en cantidades equivalentes de dulzor respecto al azúcar. El análisis sensorial se realizó con un grupo de jóvenes de ambos sexos. Se aplicó una prueba 
de aceptabilidad con escala hedónica de 5 puntos, evaluando los parámetros de aroma y sabor. Los resultados indican que el aroma de los jugos no se ve afectado por la utilización de azúcar o edulcorantes, con calificación entre 3,5 a 3,7 (que corresponde a "me gusta"). Sin embargo, en la evaluación de sabor las muestras significativamente mejor evaluadas corresponden a jugos endulzados con azúcar y sucralosa $(3,5$ y 3,6 respectivamente).

Palabras clave: análisis sensorial, endulzantes no calóricos, azúcar.

\section{BIBLIOGRAFÍA}

1. Temussi P. The Sweet Taste Receptor: A Single Receptor with Multiple Sites and Modes of Interaction. Adv Food Nutr Res. 2007;53:199-239.

2. Duran S, Rodriguez M, Cordón K, Record J. Estevia (stevia rebaudiana), edulcorante natural y no calórico. Rev Chil Nutr. 2013;39(4):203-6.

3. Shankar P, Ahuja S, Sriram K. Non-nutritive sweeteners: review and update. Nutrition. Nutrition. 2013;29:1293-9.

4. Jing MA, Bellon M, Wishart J, Young R, Blackshaw A, Jones $K$, Horowitz M, Rayner $C$. Effect of the artificial sweetener, sucralose, on gastric emptying and incretin hormone release in healthy subjects. Am J Physiol Gastrointest Liver Physiol. 2009; 296:735-9.

5. Swithers S, Martin A, Clark K, Laboy A, TL D. Body weight gain in rats consuming sweetened liquids: effects of caffeine and diet composition. Appetite. 2010;55:528-33.

6. Stellman S, Garfinkel L. Patterns of artificial sweetener use and weight change in an American Cancer Society prospective study. Appetite. 1988;11(1):85-91.

7. Fowler S, Williams $K$, Resendez $R$, Hunt $K$, Hazuda $H$, Stern M. Fueling the obesity epidemic? Artificially sweetened beverage use and long-term weight gain. Obesity. 2008;16(8):1894-900.

8. Rolls B. Effects of intense sweeteners on hunger, food intake, and body weight: a review. Am J Clin Nutr. 1991;53:872-8.

9. Benton D. Can artificial sweeteners help control body weight and prevent obesity? Nutr Res Rev. 2005;18(1):6376.

10. Bellisle F, Drewnowsk i A. Intense sweeteners, energy intake and the control of body weight. Eur J Clin Nutr. 2007;61(6):691-700.

11. Mattes $R$, Popkin B. Nonnutritive sweetener consumption in humans. Effects on appetite and food intake and their putative mechanisms. Am J Clin Nutr. 2009;89(1):1-14.

12. American Dietetic Association. Position of the American Dietetic Association: use of nutritive and nonnutritive sweeteners. J Am Diet Assoc. 2004;104(2):255-75.

13. Carpenter $R$, Lyon D, Hasdell T. Análisis Sensorial en el Desarrollo y Control de la Calidad de los Alimentos. Arancibia; 2000.

14. Lim J. Hedonic scaling: A review of methods and theory. Food Qual Prefer. 2011;22:733-47.

15. Hough G, Wakeling I, Mucci A, Chambers E, Mendez I, Rangel L. Number of consumers necessary for sensory acceptability tests. Food Qual Prefer. 2006;17:522-6.

16. Hernandez E. Evaluación Sensorial. UNIVERSIDAD NACIONAL ABIERTA Y ADISTANCIA - UNAD. Bogotá, Colombia; 2005.

17. Anzaldúa-Morales A. La evaluación sensorial de los alimentos en la teoría y la práctica. Acribia E, editor. Zaragoza, España; 1994.
18. Wittig de Penna E. Evaluación Sensorial: Una metodología actual para tecnología de alimentos [Internet]. Chile U de, editor. Santiago, Chile; 2001. Available from: http://mazinger.sisib.uchile.cl/repositorio/Ib/ciencias_quimicas_y_ farmaceuticas/wittinge01/

19. Jellinek G. Sensory Evaluation of Food. Theory and Practice. Ellis Horwood Ltd; 1985.

20. Guinard J. Sensory and consumer testing with children. Trends Food Technol. 2001;11:273-83.

21. Da Porto C, Cordaro F, Marcassa N. Effects of carbohydrate and noncarbohydrate sweeteners on the orange spirit volatile compounds. LWT- Food Sci Tech. 2006; 39: 159-65.

22. Choudhury E, Moberg P, Doty R. Influences of age and sex on a microencapsulated odor memory test. Chem Senses. 2003;28:799-805.

23. Yousem D, Maldjian J, Siddiqi F, Hummel T, Alsop D, Geckle $R$, et al. Gender effects on odor-stimulated functional magnetic resonance imaging. Brain Res. 1999;818:480-7.

24. Havlicek J, Saxton T, Roberts C, Jozifkova E, Lhota S, Valentova J, et al. He sees, she smells? Male and female reports of sensory reliance in mate choice and non-mate choice contexts. Pers Individ Dif. 2008;45:565-70.

25. Coonner M, Booth D. Preferred sweetness of a lime drink and preference for sweet over non-sweet foods, related to sex and reported age and body weight. Appetite. 1988;10(1):25-35.

26. Medeiros de Melo L, Bolini H, Efraim P. Sensory profile, acceptability, and their relationship for diabetic/reduced calorie chocolates. Food Qual Prefer. 2009;20:138-43.

27. Cadena R, Cruz A, Netto R, Castro W, Faria J, Bolini H. Sensory profile and physicochemical characteristics of mango nectar sweetened with high intensity sweeteners throughout storage time. Food Res Int. 2013;54:1670-9.

28. Cardoso J, Bolini H. Different sweeteners in peach nectar: Ideal and equivalent sweetness. Food Res Int. 2007;40:1249-53.

29. Anton S, Martin C, Han H, Coulon S, Cefalu W, Geiselman P. Effects of stevia, aspartame, and sucrose on food intake, satiety, and postprandial g/ucose and insulin levels. Appetite. 2010;55:37-43.

30. De Souza V, Pereira P, Pinheiro A, Bolini H, Borges S, Queiroz F. Analysis of various sweeteners in low-sugar mixed fruit jam: equivalent sweetness, time-intensity analysis and acceptance test. Int J Food Sci Technol. 2013;48:1541-8.

31. Liscia A, Masala C, Crnjar R, Sollai G, Solari P. Saccharin stimulates the "'deterrent'" cell in the blowfly: behavioral and electrophysiological evidence. Physiol Behav. 2004;80:637- 46.

32. Guillem-Llobat $X$. Defining, regulating and using saccharin at the outset of the industrial food era (1888-1914). Appetite. 2012;59:905-11.

33. Brito C, Bolini H. Sensory profile of sweeteners in guava nectar. Aliment Nutr. 2010;20:561-72.

34. Cardoso J, Bolini H. Descriptive profile of peach nectar sweetened with sucrose and different sweeteners. I Sens Stud. 2008;23:804-16.

35. Minsal. Encuesta Nacional de Salud ENS 2009-2010 [Internet]. 2010 [cited 2013 Oct 18]. Available from: http:// www.redsalud.gov.cl/portal/url/item/99bbf09a908d3eb8 e04001011f014b49.pdf

36. Junaeb. Situación Nutricional de Escolares de 1oBásico de Establecimientos Subvencionados del país, Situación de Obesidadpor región en Primero Básico 2011 [Internet]. 
20111 [cited 2013 Oct 18]. Available from: http://bpt. junaeb.cl:8080/MapaNutriciona/GX/

37. Kell K, Cardel M, Bohan Brown M, Fernández J. Added sugars in the diet are positively associated with diastolic blood pressure and triglycerides in children. Am J Clin Nutr. 2014; 100 (1):46-52.

38. Wang J. Consumption of added sugars and development of metabolic syndrome components among a sample of youth at risk of obesity. Appl Physiol Nutr Metab.
2014:39(4):512.

39. Johnson $R$, Nakagawa $T$, Sanchez-Lozada L, Shafiu M, Sundaram S, Le M, Ishimoto $T$, Sautin $Y$, Lanaspa $M$. . Sugar, uric acid, and the etiology of diabetes and obesity. Diabetes. 2013;62(10):3307-15.

40. Eyles H, Ni MC, Nghiem N, Blakely T. Food pricing strategies, population diets, and non-communicable disease: a systematic review of simulation studies. PLoS Med. 2012;9(12):e1001353. 